\title{
Reduced Volume of a Brainstem Substructure in Adolescents with Problematic Smartphone Use
}

\author{
In Hee Cho, Jae Hyun Yoo, Ji-Won Chun, Hyun Cho, \\ Jin-Young Kim, Jihye Choi, and Dai-Jin Kim \\ Department of Psychiatry, Seoul St. Mary's Hospital, College of Medicine, The Catholic University of Korea, Seoul, Korea
}

\begin{abstract}
Objectives: Despite the growing concern regarding the adverse effects related to problematic smartphone use (PSU), little is known about underlying morphologic changes in the brain. The brainstem is a deep brain structure that consists of several important nuclei associated with emotions, sensations, and motor functions. In this study, we sought to examine the difference in the volume of brainstem substructures among adolescents with and without PSU.

Methods: A total of 87 Korean adolescents participated in this study. The PSU group ( $n=20$, age $=16.2 \pm 1.1$, female:male $=12: 8)$ was designated if participants reported a total Smartphone Addiction Proneness Scale (SAPS) score of $\geq 42$, whereas the remaining participants were assigned to the control group ( $n=67$, age $=15.3 \pm 1.7$, female:male=19:48). High-resolution T1 magnetic resonance imaging was performed, and the volume of each of the four brainstem substructures [midbrain, pons, medulla, and superior cerebellar peduncle (SCP)] was measured. Analysis of covariance was conducted to reveal group differences after adjusting for effects of age, gender, whole brainstem volume, depressive symptoms, and impulsivity.

Results: The PSU group showed a significantly smaller volume of the SCP than the control group ( $\mathrm{F}=8.273, \mathrm{p}=0.005)$. The volume of the SCP and the SAPS score were negatively correlated (Pearson's $\mathrm{r}=-0.218, \mathrm{p}=0.047$ ).

Conclusion: The present study is the first to reveal an altered volume of the brainstem substructure among adolescents with PSU. This finding suggests that the altered white matter structure in the brainstem could be one of the neurobiological mechanisms underlying behavioral changes in PSU.
\end{abstract}

Keywords: Problematic smartphone use; Brainstem; Superior cerebellar peduncle; Structural MRI.

Received: March 23, 2021 / Revision: May 2, 2021 / Accepted: May 10, 2021

Address for correspondence: Jae Hyun Yoo, Department of Psychiatry, Seoul St. Mary's Hospital, College of Medicine, The Catholic University of Korea, 222 Banpo-daero, Seocho-gu, Seoul 06591, Korea

Tel: +82-2-2258-6773, Fax: +82-2-594-3870, E-mail: jayyoo84@gmail.com

\section{INTRODUCTION}

Since smartphones have emerged as innovative devices for mobile communication, the necessity of smartphones in our daily life has increased. Smartphones have become a core element of telecommunication and social networking, and they are actively used in daily life to acquire information, purchase goods, manage bank accounts, and enjoy hobbies.

However, such dependance on smartphone and an increase in the duration of smartphone usage has led to problems associated with excessive use. According to a meta-analysis of 31 studies that used the smartphone addiction scale, the median prevalence of problematic smartphone use (PSU) was 23.3\% [1]. Additionally, a 2019 survey on the smartphone overdependence of 28592 participants in Korea showed that

This is an Open Access article distributed under the terms of the Creative Commons Attribution Non-Commercial License (https://creativecommons.org/licenses/by-nc/4.0) which permits unrestricted non-commercial use, distribution, and reproduction in any medium, provided the original work is properly cited.
$3.8 \%$ adolescents were at a high risk of smartphone salience, loss of self-control, and problematic consequences, whereas $26.4 \%$ were at potential risk [2].

Studies have also reported that PSU is associated with negative emotional and behavioral changes. Previous studies have demonstrated that PSU is associated with symptoms of low self-esteem [3], depression [4], and anxiety [5]. Furthermore, PSU is associated with dysfunctional cognitive and behavioral changes, such as impulsivity [6] and loss of control [7]. In a recent systematic review, clinically significant levels of depression, anxiety, stress, poor quality of sleep, and academic performance [1] were reported in adolescents with PSU, suggesting serious consequences of excessive smartphone use.

Neuroimaging investigations of adolescents with PSU have started in the recent times. A recent study of participants who actively used Facebook with their smartphones showed that the number of times the participants checked Facebook and 
the total number of hours spent on Facebook was negatively correlated with the volume of the nucleus accumbens [8]. Furthermore, individuals who exhibited PSU for the purpose of communication showed a decrease in the volumes of the lateral cingulate nucleus and anterior cingulate cortex [9]. Problematic smartphone users were showed a reduced gray matter volume of the anterior insula, inferior temporal cortex, parahippocampal cortex, and anterior cingulate cortex, as well as reduced resting-state brain activity when compared to the control group [10]. A study on PSU in a Korean sample reported that the gray matter volume of the lateral orbitofrontal cortex was significantly smaller in the PSU group than in healthy controls [11]. A diffusion MRI study of PSU revealed an increase in the node centrality of the right amygdala, suggesting the involvement of the mesolimbic network [12]. Additionally, the functional connectivity between the orbitofrontal cortex and nucleus accumbens is reduced in patients with PSU [13].

The neurobiological changes in PSU have not yet converged to certain brain regions, suggesting the involvement of the fronto-striatal, salience, and fronto-limbic networks in the development of PSU. Such brain networks also play crucial roles in regulating cognition, emotions, and behavior, which is in accordance with clinically significant levels of depression, anxiety, and impulsivity in adolescents with PSU.

The brainstem is an infratentorial structure located in the middle of the cerebrum, cerebellum, and spinal cord. It receives information from the cerebellum and contains nuclei that produce serotonin, a neurotransmitter which has been observed to be reduced in depression, and dopamine, which is important for the regulation of impulsive behavior. The brainstem also plays an important role in the motor control and cognitive functions related to language, attention, and fear or pleasure responses [14]. Thus, the brainstem has been presumed to mediate emotional responses and regulate behaviors, suggesting an important role in addictive disorders. Recent animal studies have shown that a disrupted signaling of the medial frontal lobe-brainstem circuit leads to compulsive alcohol consumption [15] and that stress might induce the activation of the brainstem-reward circuit, leading to cocainerelated rewarding memories [16]. However, few studies have assessed the relationship between behavioral addiction and the changes in brainstem volume.

Among the brainstem structures, the superior cerebellar peduncle (SCP) contains bundles of nerve fibers that connect the cerebellum to the midbrain. In the midbrain, dopaminergic neurons, which play a key role in addiction, mediate neurotransmission from the ventral tegmental area and substantia nigra to the corpus striatum and limbic system [17]. The brainstem structures related to the neural input to or out- put from the midbrain may have a mediating role in addictive behaviors, via changes in the fronto-striatal and frontolimbic networks and associated neurotransmitters. In this study, we hypothesized that the volume of the brainstem would decrease according to the PSU severity. In particular, the brainstem was divided into the midbrain, pons, medulla oblongata, and SCP to assess the region that showed discrete changes according to PSU. For this purpose, we obtained structural brain magnetic resonance imaging (MRI) scans of the participants and analyzed the relationship between the structural neuroimaging data and the corresponding clinical variables, including smartphone addiction scale, depression, and impulsivity.

\section{METHODS}

\section{Participants and data collection}

Korean adolescents aged 12-18 years were recruited in this study via an online survey. Among 1631 volunteers who responded to the online questionnaire on smartphone use, 127 agreed to leave contact information for subsequent participation in the MRI study. The participants and their parents visited the laboratory to receive detailed explanations of the purpose and method of the study. The safety items for brain MRI were assessed. Eighteen adolescents could not participate in MRI study because of orthodontic appliances or claustrophobia. Among the rest of 109 participants, the imaging data of 3 participants who showed severe head movement during MRI acquisition, and 19 participants whose images were acquired using a different MRI system were further excluded. Finally, we analyzed the data of 87 participants for statistical analysis. Those who were previously diagnosed with medical (i.e., diabetes), neurological (i.e., convulsive disorder, head trauma), or psychiatric (i.e., affective disorder) disorders were excluded from the study. All the participants had normal vision and were right-handed, as assessed by the Edinburgh Handedness Inventory. This study was approved by the Institutional Review Board of Seoul St. Mary's Hospital (KC15EISI0103).

\section{Psychological assessment}

\section{Smartphone Addiction Proneness Scale}

The Smartphone Addiction Proneness Scale (SAPS) was used to distinguish the PSU group from typically developing children (TDC) [18]. This self-reported rating consists of 15 items that score from 1 to 4 points for each item (1=not at all to $4=$ =always). In a previous study, individuals with a SAPS score of $\geq 42$ points were categorized as the high-risk smartphone dependence group [18]. The same cut-off value was 
used in the current study.

\section{Beck Depression Inventory}

The underlying depressive symptoms of the study participants were evaluated using the Beck Depression Inventory (BDI), which was developed by Aaron Beck and became widely used [19]. The BDI consists of 21 items and is rated on a 4point Likert scale from 0 to 3 points $(0=$ not at all, $1=$ sometimes, 2 =often, 3 =very often) depending on the severity of the depressive symptoms.

\section{Brief Self-Control Scale}

The Brief Self-Control Scale (BSCS) is a 13-item self-reported scale developed by Tangney et al. [20]. Each item is rated on a 5 -point Likert scale ranging from 1 to 5 points ( $1=$ not at all like me, $2=$ not like me, $3=$ neutral, $4=$ like me, $5=$ =very much like me). Higher scores indicate higher self-control.

\section{Korean-Weschsler Adults Intelligence Scale}

The intellectual abilities of the participants were evaluated by a clinical psychologist using the Korean-Weschsler Adults Intelligence Scale (K-WISC-IV).

\section{Structural MRI acquisition}

Structural brain imaging data were obtained using a 3T Siemens MAGNETOM Verio system (Siemens, Erlagen, Germany) MRI equipment with a 16-channel head coil. A threedimensional T1-weighted gradient echo imaging sequence was used [176 slices, repetition time $(\mathrm{TR})=1,780 \mathrm{~ms}$, echo time $(\mathrm{TE})=2.2 \mathrm{~ms}$, image matrix $256 \times 256$ ], and structural images with $1 \times 1 \times 1 \mathrm{~mm}$ resolution were acquired. The brain structural images were analyzed using Freesurfer software (version 6.0; https://surfer.nmr.mgh.harvard.edu/). The brainstem structure segmentation technique, developed by Iglesias et al., [21] was applied to estimate the volume of the midbrain, pons, medulla oblongata, and SCP for each participant.

\section{Statistical analysis}

The demographic characteristics were analyzed using the Student's t-test and $\chi^{2}$ test according to the characteristics of each variable. The volume of each brainstem substructure may be affected by the total volume of the brainstem. Therefore, we calculated the volume of each substructure relative to the total brainstem volume and used it for further analysis. Each brainstem substructure volume was compared between the two groups with age, gender, BDI, and BSCS score as confounding variables using analysis of covariance. Subsequently, we assessed the correlation between the volume of brainstem substructures and SAPS score while adjusting for age, sex, BDI, and BSCS score. All analyses were performed using the SPSS ver. 24.0 (IBM Corp., Armonk, NY, USA), and a p-value $<0.05$ was considered statistically significant.

\section{RESULTS}

\section{Demographic characteristics of participants}

The demographic characteristics of the 87 participants (20 PSU and 67 TDC) are summarized in Table 1. There were significant differences in the demographics between the two groups, except for the intelligence level. The mean age of the PSU and TDC groups was $16.20 \pm 1.11$ and $15.27 \pm 1.69$, respectively, which was significantly lower in the TDC group $(\mathrm{t}=-2.89, \mathrm{p}=0.006)$. In addition, a significant difference in the gender ratio between the two groups $\left(\chi^{2}=6.72, \mathrm{p}=0.010\right)$ was observed. Regarding the clinical variables, the PSU group showed a significantly higher BDI $(\mathrm{t}=-3.26, \mathrm{p}=0.003)$ and SAPS scores $(\mathrm{t}=-17.42, \mathrm{p}<0.001)$ than the TDC group. Additionally, the PSU group showed a significantly lower BSCS score than the TDC group $(\mathrm{t}=4.52, \mathrm{p}<0.001)$, reflecting a lower level of self-control.

\section{Changes in the volume of brainstem substructures in PSU group}

A comparison of the brainstem substructure volume between the two groups is presented in Table 2. There was significant difference in the volume of the SCP between the PSU and TDC groups $(\mathrm{F}=8.273, \mathrm{p}=0.005)$, while those of the midbrain, pons, and medulla oblongata did not show significant differences. According to the partial correlation analysis, the volume of the SCP had a significant negative correlation with the SAPS score $(r=-0.218, p=0.047)$. However, the volumes of the midbrain $(\mathrm{r}=-0.010, \mathrm{p}=0.927)$, pons $(\mathrm{r}=0.147, \mathrm{p}=0.184)$, and medulla oblongata $(\mathrm{r}=-0.157, \mathrm{p}=0.157)$ were not correlated with the SAPS scores. The correlations between the volume of the SCP and the SAPS score for each group did not reach significance [PSU $(r=0.282, \mathrm{p}=0.289)$; TDC $(\mathrm{r}=-0.012, \mathrm{p}=0.924)]$.

Table 1. Demographic and clinical characteristics of participants

\begin{tabular}{lccc}
\hline & PSU $(n=20)$ & TDC $(n=67)$ & † or $\chi^{2}$ \\
\hline Age, yr & $16.20 \pm 1.11$ & $15.27 \pm 1.69$ & $-2.89^{* *}$ \\
Sex (male:female) & $8: 12$ & $48: 19$ & $6.72^{* *}$ \\
IQ & $100.35 \pm 16.41$ & $100.43 \pm 13.55$ & 0.85 \\
SAPS & $45.70 \pm 3.23$ & $27.39 \pm 6.26$ & $-17.42^{* * *}$ \\
BSCS & $34.40 \pm 6.55$ & $41.81 \pm 6.40$ & $4.52^{* * *}$ \\
BDI & $14.90 \pm 8.98$ & $7.97 \pm 5.65$ & $-3.26^{* *}$ \\
\hline
\end{tabular}

${ }^{* *} \mathrm{p}<0.01,{ }^{* * *} \mathrm{p}<0.001$. PSU, problematic smartphone use; TDC, typically developing children; SAPS, Smartphone Addiction Proneness Scale; BSCS, Brief Self Control Scale; BDI, Beck Depression Index 
Table 2. Comparison of brainstem structural volumes relative to the whole brainstem between problematic smartphone users and control group

\begin{tabular}{lcccc}
\hline & PSU $(\mathrm{n}=20)$ & TDC $(\mathrm{n}=67)$ & $\mathrm{F}$ & $\mathrm{P}$-value \\
\hline Midbrain, $\mathrm{mm}^{3}$ & $5888.10 \pm 552.43$ & $6113.17 \pm 602.11$ & 0.427 & 0.516 \\
Pons, $\mathrm{mm}^{3}$ & $13886.58 \pm 1843.35$ & $14062.45 \pm 1621.91$ & 0.855 & 0.358 \\
SCP, $\mathrm{mm}^{3}$ & $225.12 \pm 38.40$ & $250.92 \pm 47.45$ & 8.273 & $0.005^{* *}$ \\
Medulla, $\mathrm{mm}^{3}$ & $4197.19 \pm 502.27$ & $4280.14 \pm 434.98$ & 0.053 & 0.819 \\
Whole brainstem, $\mathrm{mm}^{3}$ & $24196.98 \pm 2805.58$ & $24706.68 \pm 2517.65$ & 0.019 & 0.892 \\
\hline
\end{tabular}

${ }^{* *} \mathrm{p}<0.01$. Analysis of covariance was performed on the volume comparison with age, sex, BSCS, and BDI as covariates. PSU, problematic smartphone use; TDC, typically developing children; SCP, superior cerebellar peduncle; BSCS, Brief Self Control Scale; BDI, Beck Depression Index

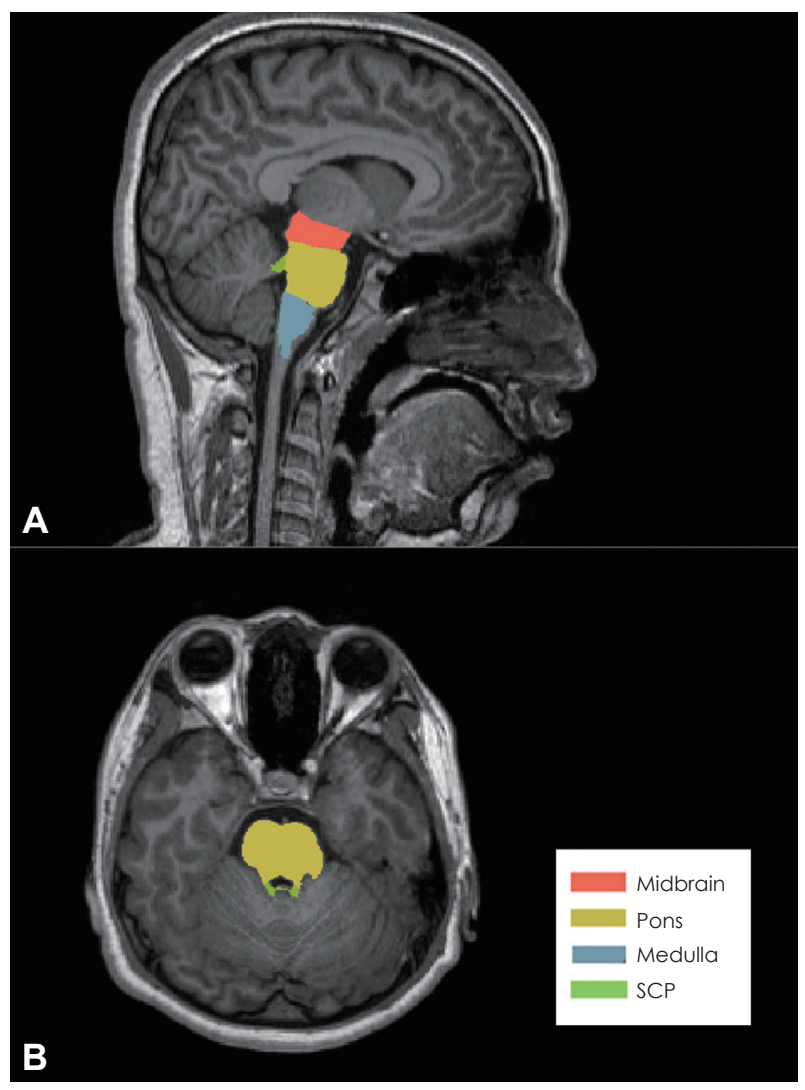

Fig. 1. Brainstem segmentation of a T1-weighted scan in (A) sagittal and (B) coronal views. SCP, superior cerebellar peduncle.

\section{DISCUSSION}

In this study, the changes in the volume of brainstem substructures were assessed in adolescents with PSU using neuroimaging and clinical variables. Our findings demonstrated that the volume of the SCP was significantly reduced, unlike that of other substructures of the brainstem (midbrain, pons, and medulla oblongata). Additionally, the severity of PSU was associated with a reduction in the volume of the SCP.

This study revealed subtle but significant changes in the volume of brainstem substructures in adolescents with PSU. Among the brainstem substructures, the midbrain, pons, and

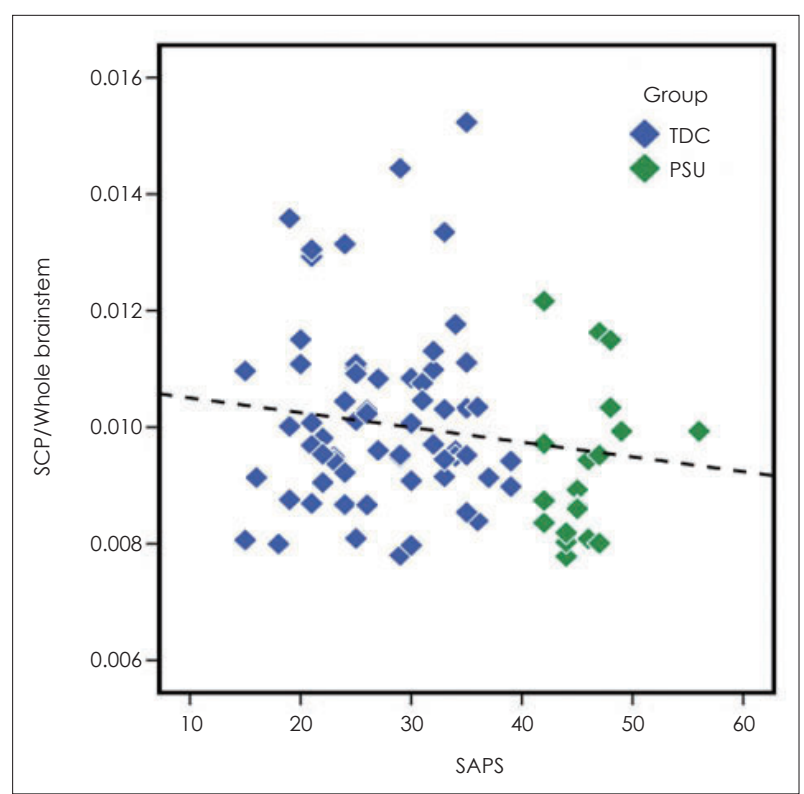

Fig. 2. Correlation between SAPS and the volume of SCP relative to the Whole brainstem. PSU, problematic smartphone use; TDC, typically developing children; SCP, superior cerebellar peduncle; SAPS, Smartphone Addiction Proneness Scale.

medulla oblongata include the major nerve nuclei and nerve fibers. In contrast, the SCP is distinct from other substructures in that it is entirely composed of nerve tracts that connect different brain regions. The SCP is located close to the fourth ventricle and filled with cerebrospinal fluid. It is possible that this anatomical location makes it susceptible to noise during MRI acquisition. Despite the susceptibility to noise from the surrounding signals, reliability in imaging can be assured because the segmentation method devised by Iglesias et al. [21] showed robust results in three different brain MRI datasets. To the best of our knowledge, this is the first study to suggest an association between behavioral addiction and changes in the volume of the brainstem structure. Notably, the volume of the SCP was significantly different after controlling for age, sex, depressive symptoms, impulsivity, and total brainstem volume. It showed a greater reduction in adolescents with more severe PSU. These findings suggest that 
changes in the brainstem may be related to the neurobiological mechanisms underlying behavioral addiction.

Previous studies on behavioral addiction have shown differences in the structure and function of the brainstem. According to one study that compared the regional homogeneity of functional MRI, the Internet addiction and control groups showed a significantly different brain synchronization pattern of the various brain regions related to sensory-motor coordination, including the brainstem [22]. Another study reported that the brainstem is one of the brain regions with enhanced activation following a stimulus or cue related to internet games in subjects with internet addiction [23]. In addition, microstructural changes in the brainstem were observed in patients with obsessive-compulsive disorder who exhibited compulsive behaviors. Additionally, the subjects with obsessive-compulsive disorder showed decreased fractional anisotropy (FA) in the right midbrain compared to the healthy control group [24]. Another study showed that the subjects with compulsive washing behaviors related to contamination had a significantly lower FA value of the white matter in the left midbrain [25].

Similar findings have been observed in studies on substance addiction. Bora and colleagues suggested changes in the connectivity of the brainstem and cerebellum with other brain regions. Diffusion tensor imaging showed that the SCP connecting the cerebellum and midbrain, and the middle cerebellar peduncle connecting the cerebellum and pons were both reduced in heroin addicts [26]. Moreover, magnetic resonance spectroscopic imaging in the cocaine and alcohol abuse groups demonstrated structural defects in the white matter of the brain. In particular, the volume of the white matter around the temporal lobe and cerebellum decreased in the cocaine abuse group [27]. These results suggest that the changes in the nerve bundles connecting the cerebellum to other brain regions via the brainstem are a common feature observed in substance and behavioral addiction, including PSU.

Up until now, only a limited number of studies have investigated the function of the SCP in the field of psychiatry. However, several research findings support the psychiatric implications of these regions. For example, those diagnosed with Asperger's syndrome had a significantly lower FA of the right SCP than the control group, and the FA of the left SCP was negatively correlated with the level of social disability [28]. The structural abnormalities of key efferent fibers of the cerebellum have been reported to affect the cerebellar feedback to the cerebrum required for adaptive social behavior [28]. Additionally, low FA of the right SCP was associated with motor function defects in children diagnosed with autism spectrum [29]. The cerebellum plays a key role in the optimal motor control by making accurate predictions through repeated learning [30]. Thus, these findings imply that the cooperative connection between the cerebellum and cerebrum through the SCP may be important for the clinical manifestation of psychiatric disorders. In this regard, the addictive behaviors shown by problematic smartphone users may be associated with aberrations in the SCP.

Koob [31] who suggested a neurobiological mechanism for addiction, reported that the addicted brain is in a disrupted state of pleasure control and motivational system. In other words, the addicted brain has lost its homeostatic modulation of reward and aversion stimuli, leading to changes in the motivational behavior. In animal studies, damage to the dentate nucleus, a deep nerve nucleus located lateral to the cerebellum, leads to a defective cerebello-thalamo-cortical pathway, causing motivational deficits [32]. Additionally, human patients with a defect in the cerebellar nucleus show difficulties in reward-based reversal learning [33]. In addition to the dopaminergic neural circuits connected in the ventral tegmental area, called the "Pleasure Center," findings from the literature suggest that the neural connections from the cerebellum may be important in behavioral addiction for an additional modulatory role as well. The SCP is a major efferent nerve pathway that originates from the deep nucleus of the cerebellum and transmits neural signals to other brain regions through the brainstem. The decreased volume of the SCP may be related to changes in the motivational processing in behavioral addiction. Therefore, the current findings might support the modulatory role of the SCP in the regulation of hedonic homeostasis, as described by Koob.

The limitations of this study are as follows. First, only a fixed number of clinical variables were assessed in the PSU and TDC groups, and other factors that could exist between the two groups could not be assessed. Even though variables, such as age, gender, depression, and impulsivity were adjusted, follow-up studies with two groups matching for these demographic variables would be necessary. Second, the area of interest for structural volume comparison was limited to the brainstem. A further assessment of the changes in the volume or function of other brain regions is needed in the future. Third, this study was based on cross-sectional data; therefore, a causal relationship between PSU and the volume of the SCP could not be established. In the future, prospective studies including children in the developmental stages would be crucial. Further research is needed to confirm any changes in the brain before and after exposure to smartphone or brain changes according to the differences in the frequency of smartphone exposure. Lastly, the PSU and TDC groups were divided based on the SAPS score of 42 . However, the severity of PSU was evaluated as a continuous variable, and a partial correlation analysis was performed in one cluster. Large-scale studies are necessary in the future 
to assess the differences in the volume of the SCP according to SAPS scores.

\section{CONCLUSION}

In this study, a significant association between PSU and a reduced volume of one of the brainstem substructures, the SCP, was revealed. This finding suggests that PSU is not just a social phenomenon, but a category of behavioral addiction related to neurobiological changes. Further evaluations of brain structures and functions in problematic smartphone users are essential to advance the understanding of PSU and develop therapeutic interventions.

\section{Availability of Data and Material}

The datasets generated or analyzed during the study are not publicly available due [REASON(S) WHY DATA ARE NOT PUBLIC] but are available from the corresponding author on reasonable request.

\section{Conflicts of Interest}

Jae Hyun Yoo, a contributing editor of the Journal of the Korean Academy of Child and Adolescent Psychiatry, was not involved in the editorial evaluation or decision to publish this article. All remaining authors have declared no conflicts of interest.

\section{Author Contributions}

Conceptualization: Jae Hyun Yoo. Data curation: Ji-Won Chun, Hyun Cho, Jihye Choi, Jin-Young Kim. Formal analysis: In Hee Cho, Jae Hyun Yoo. Funding acquisition: Jihye Choi, Dai-Jin Kim. Investigation: Ji-Won Chun, Hyun Cho, Jihye Choi. Project administration: Dai-Jin Kim. Supervision: Dai-Jin Kim. Validation: Jae Hyun Yoo. Writing-original draft: In Hee Cho. Writing_review \& editing: Jae Hyun Yoo

\section{ORCID iDs}

$\begin{array}{ll}\text { In Hee Cho } & \text { https://orcid.org/0000-0003-0694-7249 } \\ \text { Jae Hyun Yoo } & \text { https://orcid.org/0000-0002-2579-9993 } \\ \text { Ji-Won Chun } & \text { https://orcid.org/0000-0002-0629-0358 } \\ \text { Hyun Cho } & \text { https://orcid.org/0000-0003-1679-3976 } \\ \text { Jin-Young Kim } & \text { https://orcid.org/0000-0002-2996-6728 } \\ \text { Jihye Choi } & \text { https://orcid.org/0000-0002-7043-773X } \\ \text { Dai-Jin Kim } & \text { https://orcid.org/0000-0001-9408-5639 }\end{array}$

\section{Funding Statement}

This research was supported by the Basic Science Research Program through the National Research Foundation of Korea (NRF) funded by the Ministry of Science, ICT \& Future Planning (NRF2014M3C7A1062893).

\section{REFERENCES}

1) Sohn SY, Rees P, Wildridge B, Kalk NJ, Carter B. Prevalence of problematic smartphone usage and associated mental health outcomes amongst children and young people: a systematic review, meta-analysis and GRADE of the evidence. BMC Psychiatry 2019; 19:356.

2) National Information Society Agency. 2019 the survey on smartphone overdependence (report no. NIA VIII-RSE-C-19067). Dae- gu: National Information Society Agency;2019.

3) Bianchi A, Phillips JG. Psychological predictors of problem mobile phone use. Cyberpsychol Behav 2005;8:39-51.

4) Demirci K, Akgönül M, Akpinar A. Relationship of smartphone use severity with sleep quality, depression, and anxiety in university students. J Behav Addict 2015;4:85-92.

5) Matar Boumosleh J, Jaalouk D. Depression, anxiety, and smartphone addiction in university students-A cross sectional study. PLoS One 2017;12:e0182239.

6) Grant JE, Lust K, Chamberlain SR. Problematic smartphone use associated with greater alcohol consumption, mental health issues, poorer academic performance, and impulsivity. J Behav Addict 2019; 8:335-342.

7) Chen J, Liang Y, Mai C, Zhong X, Qu C. General deficit in inhibitory control of excessive smartphone users: evidence from an eventrelated potential study. Front Psychol 2016;7:511.

8) Montag C, Markowetz A, Blaszkiewicz K, Andone I, Lachmann B, Sariyska R, et al. Facebook usage on smartphones and gray matter volume of the nucleus accumbens. Behav Brain Res 2017;329:221228.

9) Montag C, Zhao Z, Sindermann C, Xu L, Fu M, Li J, et al. Internet Communication Disorder and the structure of the human brain: initial insights on WeChat addiction. Sci Rep 2018;8:2155.

10) Horvath J, Mundinger C, Schmitgen MM, Wolf ND, Sambataro F, Hirjak D, et al. Structural and functional correlates of smartphone addiction. Addict Behav 2020;105:106334.

11) Lee D, Namkoong K, Lee J, Lee BO, Jung YC. Lateral orbitofrontal gray matter abnormalities in subjects with problematic smartphone use. J Behav Addict 2019;8:404-411.

12) Tymofiyeva O, Yuan JP, Kidambi R, Huang CY, Henje E, Rubinstein ML, et al. Neural correlates of smartphone dependence in adolescents. Front Hum Neurosci 2020;14:564629.

13) Chun JW, Choi J, Cho H, Choi MR, Ahn KJ, Choi JS, et al. Role of frontostriatal connectivity in adolescents with excessive smartphone use. Front Psychiatry 2018;9:437.

14) Hurley RA, Flashman LA, Chow TW, Taber KH. The brainstem: anatomy, assessment, and clinical syndromes. J Neuropsychiatry Clin Neurosci 2010;22:iv, 1-7.

15) Siciliano CA, Noamany H, Chang CJ, Brown AR, Chen X, Leible D, et al. A cortical-brainstem circuit predicts and governs compulsive alcohol drinking. Science 2019;366:1008-1012.

16) Shinohara F, Asaoka Y, Kamii H, Minami M, Kaneda K. Stress augments the rewarding memory of cocaine via the activation of brainstem-reward circuitry. Addict Biol 2019;24:509-521.

17) Bissonette GB, Roesch MR. Development and function of the midbrain dopamine system: what we know and what we need to. Genes Brain Behav 2016;15:62-73.

18) Kim D, Lee Y, Lee J, Nam JK, Chung Y. Development of Korean smartphone addiction proneness scale for youth. PLoS One 2014; 9:e97920.

19) Beck AT, Steer RA, Brown GK. Beck depression inventory-II. San Antonio: Psychological Corporation;1996.

20) Tangney JP, Baumeister RF, Boone AL. High self-control predicts good adjustment, less pathology, better grades, and interpersonal success. J Pers 2004;72:271-324.

21) Iglesias JE, Van Leemput K, Bhatt P, Casillas C, Dutt S, Schuff N, et al. Bayesian segmentation of brainstem structures in MRI. Neuroimage 2015;113:184-195.

22) Dong G, Huang J, Du X. Alterations in regional homogeneity of resting-state brain activity in internet gaming addicts. Behav Brain Funct 2012;8:41.

23) Zhang JT, Yao YW, Potenza MN, Xia CC, Lan J, Liu L, et al. Effects of craving behavioral intervention on neural substrates of cue-induced craving in internet gaming disorder. Neuroimage Clin 2016; 12:591-599. 
24) Tao J, Wang X, Zhong Z, Han H, Liu S, Wen S, et al. Alterations of white matter fractional anisotropy in unmedicated obsessive-compulsive disorder. Neuropsychiatr Dis Treat 2017;13:69-76.

25) Lázaro L, Calvo A, Ortiz AG, Ortiz AE, Morer A, Moreno E, et al. Microstructural brain abnormalities and symptom dimensions in child and adolescent patients with obsessive-compulsive disorder: a diffusion tensor imaging study. Depress Anxiety 2014;31:10071017.

26) Bora E, Yücel M, Fornito A, Pantelis C, Harrison BJ, Cocchi L, et al. White matter microstructure in opiate addiction. Addict Biol 2012;17:141-148.

27) O'Neill J, Cardenas VA, Meyerhoff DJ. Separate and interactive effects of cocaine and alcohol dependence on brain structures and metabolites: quantitative MRI and proton MR spectroscopic imaging. Addict Biol 2001;6:347-361.

28) Catani M, Jones DK, Daly E, Embiricos N, Deeley Q, Pugliese L, et al. Altered cerebellar feedback projections in Asperger syndrome. Neuroimage 2008;41:1184-1191.

29) Hanaie R, Mohri I, Kagitani-Shimono K, Tachibana M, Azuma J, Matsuzaki J, et al. Altered microstructural connectivity of the superior cerebellar peduncle is related to motor dysfunction in children with autistic spectrum disorders. Cerebellum 2013;12:645-656.

30) Shadmehr R, Krakauer JW. A computational neuroanatomy for motor control. Exp Brain Res 2008;185:359-381.

31) Koob GF. Drug addiction: the yin and yang of hedonic homeostasis. Neuron 1996;16:893-896.

32) Bauer DJ, Kerr AL, Swain RA. Cerebellar dentate nuclei lesions reduce motivation in appetitive operant conditioning and open field exploration. Neurobiol Learn Mem 2011;95:166-175.

33) Thoma P, Bellebaum C, Koch B, Schwarz M, Daum I. The cerebellum is involved in reward-based reversal learning. Cerebellum 2008; 7:433-443. 